\title{
Response to: 'Comment on: 'How to defuse a demographic time bomb: the way forward?'
}

\author{
John C. Buchan ${ }^{1} \cdot$ Winfried Amoaku $\mathbb{1}^{2}{ }^{2} \cdot$ Beth Barnes $^{3} \cdot$ Andy Cassels-Brown ${ }^{4} \cdot$ Bernard Y. Chang ${ }^{4} \cdot$ Jane Harcourt ${ }^{5}$. \\ Darren Shickle $^{6} \cdot$ Anne Fiona Spencer $^{7} \cdot$ Stephen A. Vernon $^{8} \cdot$ Carrie MacEwen $^{9}$
}

Received: 13 December 2017 / Accepted: 14 December 2017 / Published online: 9 February 2018

(c) The Royal College of Ophthalmologists 2018

We thank Professor Claoué for his letter regarding Immediate Sequential Bilateral Cataract Surgery (ISBCS).

Given the increasing mismatch between resources and demand, the national need to move to more efficient working practices has to be an inclusive, incremental, collective effort. By advocating a leap to a two-visit bilateral cataract pathway or any other unconventional efficiency measure, we would be concerned that we might lose the ear of the majority of departments whose pathway currently involves five visits for bilateral cataracts.

The October 2017 NICE guidelines [NG77] on adult cataract surgery recommend us to 'Consider bilateral simultaneous cataract surgery for people who are at low risk of complications during and after surgery'. While there was enough evidence to permit NICE to come to this conclusion, a recent systematic review and meta-analysis of ISBCS described the quality of evidence for this procedure as 'low to very-low' [1], and the very fact that ISBCS has been repeatedly proposed as a safe practice for over three decades [2, 3], but has gained little traction

John C. Buchan

john.buchan@1shtm.ac.uk

1 International Centre for Eye Health, London School of Hygiene and Tropical Medicine, London, UK

2 Academic Ophthalmology, 'B' Floor, Eye and ENT Centre, University Hospital QMC, University of Nottingham, Nottingham, UK

3 Head of Professional Support, The Royal College of Ophthalmologists, London, UK

4 Department of Ophthalmology, St James's University Hospital, Leeds, UK

5 Eye Department, Aberdeen Royal Infirmary, Forresterhill, Aberdeen, UK

6 Leeds Institute of Health Sciences, Level 10, Worsley Building, University of Leeds, Leeds, UK

7 Manchester Royal Eye Hospital, Manchester, UK

8 The BMI Park Hospital, Arnold, Nottingham, UK

9 Ward 25, Ninewells Hospital, Dundee, UK in the UK suggests that more work is needed to convince those providing cataract surgery to routinely offer ISBCS.

Prof Claoue expresses the belief that there is little risk from ISBCS, however, intuitively ophthalmic surgeons find this hard to comprehend [4]; the very-low risk of bilateral endophthalmitis notwithstanding, consideration might be given to the risk of bilateral cystoid macular oedema (CMO). Even excluding patients with risk factors the rate of clinical CMO in the largest study in the UK to date with 35563 low risk eyes was $1.17 \%$ [5], and first eye CMO is strongly predictive of second eye $\mathrm{CMO}$ with genetic factors at play [6], a risk that may not be amenable to reduction by topical NSAIDs in these low risk eyes [7].

The key to promoting ISBCS nationally is unlikely to lie in convincing surgeons that there is a lack of risk. The opportunity to save money for the NHS is also a poor motivator. Rather, we should purpose to demonstrate that there is the possibility of doing good to potentially tens of thousands of patients annually by providing faster visual rehabilitation and better quality of life through ISBCS. Evidence of improved quality of life would perhaps be more convincing to ophthalmologists, but would require a large national randomised controlled trial.

Thus convinced, surgeons would be able to enter into an honest and informed process of shared decision making with patients - some of whom will doubtless be delighted with the potential benefits of ISBCS, and some of whom will doubtless be wary of the potential risks, small as they are.

\section{Compliance with ethical standards}

Conflict of interest The authors declare that they have no competing interests.

\section{References}

1. Kessel L, Andresen J, Erngaard D, Flesner P, Tendal B, Hjortdal J. Immediate sequential bilateral cataract surgery: a systematic review and meta-analysis. J Ophthalmol. 2015;2015:912481.

2. Smith GT, Liu CS. Is it time for a new attitude to "simultaneous" bilateral cataract surgery? Br J Ophthalmol. 2001;85:1489-96. 
3. Fenton PJ, Gardner ID. Simultaneous bilateral intraocular surgery. Trans Ophthalmol Soc UK. 1982;102:298-301.

4. Schachat AP. Simultaneous bilateral endophthalmitis after immediate sequential bilateral cataract surgery: what's the risk of functional blindness? Am J Ophthalmol. 2014;158:410-1.

5. Chu CJ, et al. Risk Factors and Incidence of Macular Edema after Cataract Surgery A Database Study of 81984 Eyes. Ophthalmology. 2016;123:316-323.
6. Masood I, Negi A, Vernon SA, et al. The $-174 \mathrm{G} / \mathrm{C}$ interleukin-6 promoter polymorphism influences the development of macular oedema following uncomplicated phacoemulsification surgery. Eye. 2007;21:1412-5.

7. McCafferty S, et al. Pseudophakic cystoid macular edema prevention and risk factors; prospective study with adjunctive once daily topical nepafenac $0.3 \%$ versus placebo. BMC Ophthalmol. 2017;17:16. 20

\title{
Foetal haemoglobin, blood transfusion, and retinopathy of prematurity
}

\author{
Luciana Teofili $\mathbb{D}^{1} \cdot$ Maria Bianchi ${ }^{1} \cdot$ Antonio Baldascino $^{2} \cdot$ Patrizia Papacci $^{3} \cdot$ Giovanni Vento $^{3}$
}

Received: 3 September 2017 / Accepted: 1 November 2017 / Published online: 12 February 2018

(c) The Royal College of Ophthalmologists 2018

Stutchfield et al. have recently demonstrated that low foetal haemoglobin $(\mathrm{HbF})$ levels predict retinopathy of prematurity (ROP) [1]. There is an increasing awareness that red blood cell (RBC) transfusions are independent risk factors for all prematurity-associated diseases (PAD) [2]. Since adult haemoglobin $(\mathrm{HbA})$ releases oxygen more efficiently than $\mathrm{HbF}$, autologous cord blood (CB) transfusion has been attempted, with limited results due to the low volume of $\mathrm{CB}$ collected [3]. We have shown that allogeneic $\mathrm{CB}$ RBC concentrates obtained from healthy full-term babies can fulfil transfusion requirements of preterm neonates (PNs) with gestational age $\leq 30$ weeks and/or birth weight $\leq 1500 \mathrm{~g}$, in their first 28 days of life [4]. At first transfusion episode, $\mathrm{PNs}$ received $\mathrm{ABO}-\mathrm{Rh}(\mathrm{D})$ matched CB-RBCs if available, or adult RBCs if CB units were not available. At subsequent transfusions, the same regimen was adopted, unless CB-RBCs were unavailable. Overall, 9 patients received CB-RBCs and 11 adult-RBCs; 6 patients ( 3 in each group) died before ROP assessment. Table 1 illustrates ROP findings in 14 surviving patients. All PNs

Luciana Teofili

luciana.teofili@unicatt.it

1 Transfusion Medicine Department, Fondazione Policlinico Universitario A. Gemelli-Università Cattolica del Sacro Cuore, Roma, Italy

2 Ophtalmology Department, Fondazione Policlinico Universitario A. Gemelli-Università Cattolica del Sacro Cuore, Roma, Italy

3 Neonatal Intensive Care Unit, Fondazione Policlinico Universitario A. Gemelli-Università Cattolica del Sacro Cuore, Roma, Italy receiving adult-RBCs developed ROP, while two of six patients in the CB-RBC group did not. Stage 3 ROP was observed in four heavily transfused extremely PNs: three of them were transfused only or mainly with adult-RBCs (patients 8,10 and 14, respectively; Table 1).

Transfusions contribute to the overwhelming oxidative burden caused by infections, oxygen therapy and inflammatory diseases in PNs. Unfortunately, to monitor in these patients lipid peroxidation products or other biomarkers of the oxidative stress, requires sophisticated methodologies and exceeding volume of biologic samples. Hence, these investigations are so far confined to the research field [5]. In this regard, the study of Stutchfield et al. suggests that monitoring $\mathrm{HbF}$ levels in PNs might be a feasible and reliable tool to figure out to what extent transfusions might favour PAD development.

Acknowledgements This study was supported by Genitin Onlus (Associazione Genitori Bambini Prematuri), Rome, Italy.

\section{Compliance with ethical standards}

Conflict of interest The authors declare that they have no competing interests.

\section{References}

1. Stutchfield CJ, Jain A, Odd D, Williams C, Markham R. Foetal haemoglobin, blood transfusion, and retinopathy of prematurity in very preterm infants: a pilot prospective cohort study. Eye (Lond). 2017;31:1451-1455. 